\title{
Peptidoglycan $N$-acetylglucosamine deacetylation decreases autolysis in Lactococcus lactis
}

Correspondence

Marie-Pierre Chapot-Chartier

Marie-Pierre.Chapot@jouy.inra.fr

Received 5 January 2007

Revised 24 May 2007

Accepted 22 June 2007

\author{
Mickael Meyrand, Aïda Boughammoura, † Pascal Courtin, \\ Christine Mézange, Alain Guillot and Marie-Pierre Chapot-Chartier
}

INRA, Unité de Biochimie Bactérienne, UR477, 78350 Jouy-en-Josas, France

\section{INTRODUCTION}

Peptidoglycan is the major cell wall component of Grampositive bacteria, and ensures the stability and rigidity of the cell wall (Delcour et al., 1999). During growth, bacteria synthesize enzymes capable of hydrolysing their own peptidoglycan. These peptidoglycan hydrolases (PGHs) are required for cell separation after division and are involved in several other cellular processes that require peptidoglycan remodelling, such as peptidoglycan turnover and cell wall expansion (Smith et al., 2000). Under conditions which result in cessation of peptidoglycan synthesis, such as stationary phase or exposure to antibiotics, PGH activity may cause cellular autolysis, leading to the release of intracellular components.

Since PGHs are potentially lethal enzymes and are present in the cells during bacterial growth, it is usually assumed that their activities are regulated at the post-translational level (Shockman \& Höltje, 1994; Smith et al., 2000). Different mechanisms of PGH control have been proposed, including proteolytic maturation (Buist et al., 1998; Poquet et al., 2000; Shockman, 1992), interaction with a specific modifier protein (Lazarevic et al., 1992), regulation by the membrane proton motive force (Calamita et al., 2001;

†Present address: Laboratoire des Interactions Plantes-Pathogènes, Institut National Agronomique de Paris-Grignon, 16 rue Claude Bernard, 75231 Paris Cedex 05, France.

Abbreviations: $\mathrm{PGH}$, peptidoglycan hydrolase; GlcNAc, $\mathrm{N}$-acetylglucosamine; MurNAc, $\mathrm{N}$-acetylmuramic acid; SCO, single crossing-over; PSD, post-source decay; PMF, peptide mass fingerprinting.
Kemper et al., 1993), and interaction with secondary cell wall polymers such as teichoic acids (Palumbo et al., 2006; Wecke et al., 1997). Finally, structural modifications of the peptidoglycan substrate by $\mathrm{O}$-acetylation (Pfeffer et al., 2006) or de- $N$-acetylation (Atrih et al., 1999) may control the action of PGHs.

The peptidoglycan hydrolase complement of Lactococcus lactis, the model Gram-positive lactic acid bacterium, comprises five PGHs, including three $\mathrm{N}$-acetylglucosaminidases (Buist et al., 1995; Huard et al., 2004, 2003) and one $\gamma$ D-glutaminyl-L-lysyl-endopeptidase (Redko et al., 2007). The autolysin AcmA is involved in cell separation and is the major effector of cellular autolysis in stationary phase (Buist et al., 1995). The AcmA protein has two domains: an $\mathrm{N}$-terminal catalytic domain endowed with $\mathrm{N}$-acetylglucosaminidase specificity (Steen et al., 2005a), and a C-terminal domain with three LysM modules involved in cell wall binding and which recognize peptidoglycan (Steen et al., 2003).

Our objective was to examine the impact of structural modifications of peptidoglycan on $L$. lactis cellular autolysis and on the activity of AcmA autolysin in the cells. Peptidoglycan consists of glycan strands made of alternating $\beta$-1,4-linked $N$-acetylglucosamine (GlcNAc) and $\mathrm{N}$-acetylmuramic acid (MurNAc) which are crosslinked by short peptide chains. Its structure can be analysed by identification of the constituent muropeptides obtained after muramidase digestion. Recently, we performed a detailed analysis of $L$. lactis peptidoglycan structure and observed several deacetylated muropeptides (Courtin et al., 
2006). A gene $(x y n D)$ encoding a putative peptidoglycan deacetylase was identified in the L. lactis IL1403 genome on the basis of sequence similarity with the peptidoglycan GlcNAc deacetylase PgdA from Streptococcus pneumoniae (Vollmer \& Tomasz, 2000). These peptidoglycan deacetylases belong to the carbohydrate esterase family 4 (CE4), which also includes chitin deacetylases, acetylxylan esterases and chitooligosaccharide deacetylase [Psylinakis et al., 2005; Carbohydrate Active Enzymes database (http:// www.cazy.org/); Coutinho \& Henrissat, 1999].

In this study, we show that L. lactis $x y n D$ (renamed $p g d A$ ) encodes a peptidoglycan GlcNAc deacetylase. In addition, we show that an increase in the degree of peptidoglycan de$\mathrm{N}$-acetylation results in decreased autolysis of L. lactis cells, due to a reduced susceptibility of deacetylated peptidoglycan to the major autolysin AcmA.

\section{METHODS}

Bacterial strains, plasmids and growth conditions. The bacterial strains and plasmids used in this study are listed in Table 1. Escherichia coli strains were grown at $37^{\circ} \mathrm{C}$ in Luria-Bertani (LB; Difco) medium with shaking. L. lactis strains were grown in M17 medium (Difco) supplemented with $0.5 \%(\mathrm{w} / \mathrm{v})$ glucose at $30{ }^{\circ} \mathrm{C}$. Growth and autolysis were monitored by measuring $\mathrm{OD}_{600}$ with a spectrophotometer (Uvikon XL, Bio-Tek Instruments). Plasmids were selected by addition of antibiotics as follows: for $L$. lactis, erythromycin $\left(5 \mu \mathrm{g} \mathrm{ml}^{-1}\right)$, chloramphenicol $\left(5 \mu \mathrm{g} \mathrm{ml}^{-1}\right)$; for E. coli, ampicillin $\left(50 \mu \mathrm{g} \mathrm{ml}^{-1}\right)$, kanamycin $\left(50 \mu \mathrm{g} \mathrm{ml}^{-1}\right)$, chloramphenicol $\left(10 \mu \mathrm{g} \mathrm{ml}^{-1}\right)$.

General recombinant DNA techniques. Molecular cloning techniques were performed using standard procedures (Sambrook et al., 1989). Restriction enzymes (New England Biolabs), T4 DNA ligase (Epicenter), Taq DNA polymerase (Qbiogen) and the TripleMaster PCR System (Eppendorf) were used as recommended by the manufacturers. Oligonucleotides were purchased from Invitrogen. Plasmids were extracted using the QIAprep Spin Miniprep kit (Qiagen). PCR was performed with a GeneAmp 2400 PCR system (Perkin-Elmer). DNA sequences were determined with an Applied Biosystems 370A automated DNA sequencer, and with ABI PRISM dye terminator cycle sequencing and dye primer cycle sequencing kits (Perkin-Elmer). Preparation of competent cells and electrotransformation of L. lactis were carried out as described elsewhere (Holo \& Nes, 1989).

Construction of the pgdA mutant and complementation of the mutant. The gene $p g d A(x y n D)$ was inactivated in L. lactis strain IL6288 by single crossing-over (SCO) plasmid integration. An internal $p g d A$ fragment was PCR-amplified with primers $5^{\prime}$ TACCTTGCTTATAGGAGCG- ${ }^{\prime}$ and $5^{\prime}$-CAGGCTTGGGCTTGTTTTC-3', using IL1403 DNA as template. The resulting $597 \mathrm{bp}$ fragment was cloned into the pGEM-T Easy vector and sequenced. It was then cloned into the NcoI and SacI restriction sites of the pJIM2242

Table 1. Bacterial strains and plasmids used in this study

\begin{tabular}{|c|c|c|}
\hline Strains and plasmids & Relevant genotype or phenotype & Source or reference \\
\hline \multicolumn{3}{|l|}{ E. coli strains } \\
\hline TG1 & supE hsd $\Delta 5$ thi $\Delta\left(\right.$ lac-proAB) $\mathrm{F}^{\prime}\left[\operatorname{traD} 36\right.$ proAB $^{+}$lacl ${ }^{\mathrm{q}}$ lacZ $\left.\Delta \mathrm{M} 15\right]$ & \\
\hline BL21(DE3) & $\mathrm{F}^{-} \operatorname{omp} \mathrm{T} h s d \mathrm{~S}_{\mathrm{B}}\left(\mathrm{r}_{\mathrm{B}}^{-} \mathrm{m}_{\mathrm{B}}^{-}\right)$gal $d c m$ bearing $\mathrm{T} 7 \mathrm{RNA}$ polymerase gene ( $\lambda \mathrm{DE} 3$ lysogen) & Studier \& Moffatt (1986) \\
\hline BL21(DE3)pLysE & $\begin{array}{l}\mathrm{Cm}^{\mathrm{r}} \text {, BL21(DE3) containing pLysE plasmid producing T7 lysozyme, an inhibitor } \\
\text { of T7 RNA polymerase }\end{array}$ & Invitrogen \\
\hline TIL933 & $\mathrm{Cm}^{\mathrm{r}} \mathrm{Ap}^{\mathrm{r}} \mathrm{Kan}^{\mathrm{r}}$, BL21(DE3)pLysE derivative containing pET11a-Kan & This study \\
\hline TIL934 & $\begin{array}{l}\mathrm{Cm}^{\mathrm{r}} \mathrm{Ap}^{\mathrm{r}} \mathrm{Kan}^{\mathrm{r}} \text {, BL21(DE3)pLysE derivative expressing AcmA with hexa-His-tag } \\
\text { with pET11a-Kan plasmid }\end{array}$ & This study \\
\hline \multicolumn{3}{|l|}{ L. lactis strains } \\
\hline IL1403 & Plasmid-free strain & Chopin et al. (1984) \\
\hline IL6288 & IL1403 derivative cured of its six prophages & M.-C. Chopin* \\
\hline TIL926 & $\begin{array}{l}\mathrm{Em}^{\mathrm{r}} \text {, IL6288 derivative, } p g d A \text { mutant obtained by SCO integration of pJIM2242 } \\
\text { plasmid containing a } 597 \text { bp } p g d A \text { internal fragment }\end{array}$ & This study \\
\hline TIL926(pJIMpgdA) & $\mathrm{Em}^{\mathrm{r}} \mathrm{Cm}^{\mathrm{r}}$, TIL926 derivative containing pJIM $p g d A$ plasmid & This study \\
\hline TIL926(pJIM) & $\mathrm{Em}^{\mathrm{r}} \mathrm{Cm}^{\mathrm{r}}$, TIL926 derivative containing pJIM2246 & This study \\
\hline PA1001(pPA3) & Derivative of NZ9000 lacking $a c m A$ and $h t r A$ and containing pPA3 plasmid & Bosma et al. (2006) \\
\hline MG1363acmA $\Delta 1$ & Derivative of MG1363 carrying an internal deletion in $\mathrm{acmA}$ & Buist et al. (1995) \\
\hline \multicolumn{3}{|l|}{ Plasmids } \\
\hline pGEM-T Easy & $\mathrm{Ap}^{\mathrm{r}}$, cloning vector with $\mathrm{T}$ overhangs & Promega \\
\hline pET11a & $\mathrm{Ap}^{\mathrm{r}}$, expression vector with $\mathrm{T} 7$ lac promoter & Novagen \\
\hline pET11a-Kan & $\mathrm{Ap}^{\mathrm{r}} \mathrm{Kan}^{\mathrm{r}}$, pET11a derivative with kanamycin-resistance cassette cloned into ClaI site & Chich et al. (1995) \\
\hline pJIM2242 & $\mathrm{Em}^{\mathrm{r}}$, derivative of pORI28 non-replicative vector in L. lactis & Guedon et al. (2001) \\
\hline pJIM2246 & $\mathrm{Cm}^{\mathrm{r}}$, high-copy lactococcal cloning vector & Renault et al. (1996) \\
\hline pJIMpgdA & $\mathrm{Cm}^{\mathrm{r}}$, pJIM2246 derivative carrying $p g d A$ gene under control of its own promoter & This study \\
\hline pPA3 & $\begin{array}{l}\mathrm{Cm}^{\mathrm{r}} \text {, pNZ8048 derivative containing c-myc, } 3^{\prime} \text { end of acmA (nt 835-1492) } \\
\text { under control of nisin-inducible promoter and usp45 signal sequence }\end{array}$ & Bosma et al. (2006) \\
\hline
\end{tabular}

${ }^{\star}$ INRA, Génétique Microbienne, Jouy-en-Josas, France. 
plasmid vector, a non-replicative plasmid in L. lactis. The resulting plasmid was produced in E. coli TG1 and used to transform L. lactis IL6288. Integration of this plasmid by SCO into the IL6288 chromosome was performed with helper plasmid pGhost8, as described elsewhere (Guedon et al., 2001). Erythromycin-resistant clone TIL926 was selected. Plasmid integration was verified by PCR and Southern blot hybridization.

Complementation of the IL6288 pgdA-negative mutant was obtained by cloning the $p g d A$ gene into the pJIM2246 multicopy plasmid vector. A $1351 \mathrm{bp}$ DNA fragment encoding $p g d A$ with its putative promoter and terminator was amplified by PCR from IL1403 total DNA with primers $5^{\prime}$-AAACTGCAGGGATATGTTAAGATAGGAGG-3' (PstI site underlined) and 5'-ATATGCGGCCGCTCAAGTTTCCCATAAGTTGTC-3' (NotI site underlined). The fragment was digested with PstI and NotI and cloned into a PstI/NotI-linearized pJIM2246 vector. The resulting plasmid was produced in E. coli TG1 and used to transform TIL926. The resulting strain was named TIL926(pJIM $p g d A$ ). A negative control strain was obtained by transformation of TIL926 with pJIM2246 empty plasmid.

Cell wall peptidoglycan preparation. Peptidoglycan from L. lactis strains was prepared as described previously (Courtin et al., 2006). Cells from a $500 \mathrm{ml}$ exponentially growing culture $\left(\mathrm{OD}_{600} 0.3\right)$ were chilled on ice and harvested by centrifugation $\left(4200 \mathrm{~g}, 10 \mathrm{~min}, 4{ }^{\circ} \mathrm{C}\right)$. Cells were suspended in $20 \mathrm{ml}$ deionized $\mathrm{H}_{2} \mathrm{O}$ and boiled for $10 \mathrm{~min}$. They were then resuspended in $1 \mathrm{ml} 5 \%$ (w/v) SDS and boiled for $25 \mathrm{~min}$. The pellet obtained by centrifugation at $20000 \mathrm{~g}$ for $10 \mathrm{~min}$ was resuspended in $1 \mathrm{ml} 4 \%(\mathrm{w} / \mathrm{v})$ SDS and boiled again for $15 \mathrm{~min}$. Cell walls were recovered by centrifugation at $20000 \mathrm{~g}$ for $10 \mathrm{~min}$ and washed six times with deionized $\mathrm{H}_{2} \mathrm{O}$ to remove SDS. To eliminate proteins, the cell wall pellet was treated with Pronase $\left(2 \mathrm{mg} \mathrm{ml}^{-1}\right)$ for $90 \mathrm{~min}$ at $60{ }^{\circ} \mathrm{C}$, then by trypsin $\left(200 \mu \mathrm{g} \mathrm{ml}^{-1}\right)$ for $16 \mathrm{~h}$ at $37^{\circ} \mathrm{C}$. The pellet containing peptidoglycan was treated with $48 \%$ hydrofluoric acid overnight at $4{ }^{\circ} \mathrm{C}$ to eliminate teichoic acids, washed twice with $0.25 \mathrm{M}$ Tris/ $\mathrm{HCl}, \mathrm{pH}$ 8.0, and then four times with deionized $\mathrm{H}_{2} \mathrm{O}$. The final pellet was freeze-dried and stored at $-20{ }^{\circ} \mathrm{C}$.

Structural analysis of $\boldsymbol{L}$. lactis peptidoglycan. Purified peptidoglycan $(4 \mathrm{mg}$ dry weight in $500 \mu \mathrm{l})$ was digested with mutanolysin

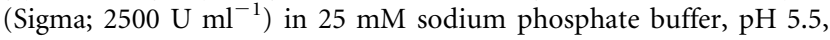
for $19 \mathrm{~h}$ at $37^{\circ} \mathrm{C}$ under rotational shaking. The enzyme was inactivated by boiling the sample for $3 \mathrm{~min}$ and insoluble material was removed by centrifugation. The soluble muropeptides were reduced with sodium borohydride and then separated by reversedphase HPLC using a Hypersil ODS column (C18; $250 \times 4.6 \mathrm{~mm}$ internal diameter; $5 \mu \mathrm{m}$ particle size; Thermo Hypersil-Keystone) at $50{ }^{\circ} \mathrm{C}$ using ammonium phosphate buffer and a methanol linear gradient, as described previously (Courtin et al., 2006). Peaks were analysed without desalting by MALDI-TOF MS with a Voyager DE STR mass spectrometer (Applied Biosystems), as described previously (Courtin et al., 2006).

For MALDI-post-source decay (PSD) analysis (Chaurand et al., 1999), the purified muropeptides were desalted by HPLC on a Betasil C18 column $(250 \times 4.6 \mathrm{~mm}$ internal diameter; $5 \mu \mathrm{m}$ particle size; Thermo Hypersil-Keystone) equilibrated with solvent $\mathrm{A}\left[\mathrm{H}_{2} \mathrm{O}\right.$ with $0.115 \%$ trifluoroacetic acid (TFA)] at $50{ }^{\circ} \mathrm{C}$. The muropeptides were eluted with a linear gradient from 0 to $33 \%$ of solvent B $(60 \%$ acetonitrile with $0.1 \%$ TFA) in $40 \mathrm{~min}$. The desalted muropeptides were then dried with a SpeedVac concentrator, and resuspended in $10 \mu \mathrm{l} 50 \%$ acetonitrile for MALDI-PSD analysis.

Expression and purification of His-tagged AcmA protein in $E$. coli. The major autolysin AcmA from L. lactis IL1403 was expressed in E. coli BL21(DE3)pLysE without its putative signal sequence and with a C-terminal hexa-His-tag. With the primers AUT1 (5'-GGAATTCCATATGGCAACCAATTCCACAGAA-3'; NdeI site underlined) and AUT4 (5'-CGGGATCCTTGTGATGGTGATGGTGATGTTTAATACGAAGATATT $\overline{\text { GACCAAT }}-3^{\prime}$; BamHI site underlined; encoding a hexa-His-tag) selected from the IL1403 genome sequence, a $1145 \mathrm{~kb}$ DNA fragment was PCR-amplified from L. lactis IL1403 DNA. This fragment was cloned into the expression vector pET-11a-Kan (Chich et al., 1995) under the control of the T7 promoter with the lac operator. The resulting plasmid was produced in E. coli TG1 and used to transform E. coli BL21(DE3)pLysE. The resulting strain was named TIL934. To induce expression of recombinant hexa-His-tagged AcmA, IPTG was added at a final concentration of $1 \mathrm{mM}$ to the culture at an $\mathrm{OD}_{600}$ of 0.6 . The control strain BL21(DE3)pLysE containing the empty pET11a-Kan vector (TIL933) was treated in a similar way. Bacteria were grown at $37^{\circ} \mathrm{C}$ until IPTG addition and were then transferred to $30{ }^{\circ} \mathrm{C}$ during the expression time $(3 \mathrm{~h})$ to avoid inclusion body formation. Cells were harvested by centrifugation and disrupted by one passage at a pressure of 1600 bar with a Basic Z Cell Disruption System (Constant Systems). The soluble fraction containing the recombinant protein was recovered after centrifugation at $15000 \mathrm{~g}$ for $15 \mathrm{~min}$ at $4{ }^{\circ} \mathrm{C}$. The hexa-His-tagged protein was purified on a His-Trap FF column $(1 \mathrm{ml}$, Amersham Biosciences) according to the manufacturer's instructions using a fast protein liquid chromatography system (Amersham Biosciences). Elution was carried out with an imidazole concentration gradient (20-500 $\mathrm{mM}$ in $15 \mathrm{~min})$. Fractions were collected and analysed by SDS-PAGE and zymogram. Fractions containing AcmA eluted at an imidazole concentration between 300 and $400 \mathrm{mM}$.

SDS-PAGE and renaturing SDS-PAGE (zymogram). SDS-PAGE was performed with $10 \%(\mathrm{w} / \mathrm{v})$ polyacrylamide separating gels. Renaturing SDS-PAGE was performed as previously described (Huard et al., 2003). The polyacrylamide gels contained $0.2 \%(\mathrm{w} / \mathrm{v})$ Micrococcus luteus ATCC 4698 (Sigma) or $0.4 \%$ (w/v) L. lactis autoclaved cells, or $0.08 \%(\mathrm{w} / \mathrm{v})$ L. lactis peptidoglycan as enzyme substrates. After sample migration in the gels, the gels were washed for $30 \mathrm{~min}$ in deionized $\mathrm{H}_{2} \mathrm{O}$ at room temperature and then incubated in $50 \mathrm{mM}$ Tris/ $\mathrm{HCl}, \mathrm{pH} 7.0$, containing $0.1 \%(\mathrm{v} / \mathrm{v})$ Triton X-100, overnight at $37{ }^{\circ} \mathrm{C}$. The gels were subsequently washed for $30 \mathrm{~min}$ in deionized $\mathrm{H}_{2} \mathrm{O}$, then stained with $0.1 \%$ Methylene Blue in $0.01 \%(\mathrm{w} / \mathrm{v}) \mathrm{KOH}$ for $2 \mathrm{~h}$ at room temperature and destained in deionized $\mathrm{H}_{2} \mathrm{O}$. Gel images were generated with a DuoScan T1200 scanner (Agfa-Gevaert).

Protein identification by peptide mass fingerprinting (PMF). Proteins were digested with trypsin and the masses of tryptic peptides were analysed by MALDI-TOF MS, as described previously (Guillot et al., 2003), with a Voyager DE STR instrument (Applied Biosystems) by the Plateau d'Analyses Protéomiques par Séquençage et Spectrométrie de Masse (PAPSS) at INRA, Jouy-en-Josas. Database searches were conducted with the MS-Fit software (http://prospector.ucsf.edu) either on an $L$. lactis-specific database containing protein sequences deduced from the genome sequence of L. lactis IL1403 or on the SWISS-PROT database.

Triton X-100-induced autolysis in buffer solution. L. lactis strains were grown in M17 medium to mid-exponential phase $\left(\mathrm{OD}_{600} 0.8\right)$. Cells were harvested by centrifugation at $5000 \mathrm{~g}$ for $10 \mathrm{~min}$ at $4{ }^{\circ} \mathrm{C}$, washed once with $50 \mathrm{mM}$ potassium phosphate buffer, $\mathrm{pH} 7.0$, and resuspended at an $\mathrm{OD}_{600}$ of 1 in $50 \mathrm{mM}$ potassium phosphate buffer, pH 7.0, supplemented with $0.05 \%$ Triton X-100 (Cornett \& Shockman, 1978). Cell suspensions were then transferred into 100well sterile microplates and incubated at $30{ }^{\circ} \mathrm{C}$. Autolysis was monitored by measuring the $\mathrm{OD}_{600}$ of the cell suspensions with an automated incubator/optical density reader (Microbiology Workstation Bioscreen C, Labsystems).

Assay of AcmA activity in L. lactis autoclaved cells. L. lactis autoclaved cells were used as a substrate for measuring AcmA activity 
and were prepared as follows. L. lactis strains were grown in M17 medium up to the end of exponential phase. Cells were harvested by centrifugation at $5000 \mathrm{~g}$ for $15 \mathrm{~min}$ at $4{ }^{\circ} \mathrm{C}$, washed once with $10 \mathrm{mM}$ Tris/ $\mathrm{HCl}, \mathrm{pH} 7.5$, at $4{ }^{\circ} \mathrm{C}$, washed once with deionized $\mathrm{H}_{2} \mathrm{O}$, and freeze-dried. The dried samples were resuspended in deionized $\mathrm{H}_{2} \mathrm{O}$ at $10 \%(\mathrm{w} / \mathrm{v})$, autoclaved and stored at $4{ }^{\circ} \mathrm{C}$.

Autoclaved cells were diluted in $25 \mathrm{mM}$ Tris/ $\mathrm{HCl}, \mathrm{pH} 7$, to $\mathrm{OD}_{600}$ 0.5 . Purified hexa-His-tagged AcmA $\left(\sim 1.5 \mu \mathrm{g} \mathrm{ml}{ }^{-1}\right.$ final concentration) was added in a final volume of $1 \mathrm{ml}$, and the $\mathrm{OD}_{600}$ of the cell suspension was monitored with a spectrophotometer (Uvikon XL, Bio-Tek Instruments). The extent of autolysis was expressed as the percentage decrease in $\mathrm{OD}_{600}$.

Binding of c-Myc-PA fusion protein to peptidoglycan. The cMyc-PA (PA3) fusion protein corresponds to the cell wall binding domain of AcmA (PA; C-terminal 218 aa of AcmA) fused to a c-Myc epitope at its $\mathrm{N}$ terminus (Bosma et al., 2006). It was produced in the supernatant of strain L. lactis PA1001(pPA3) (a kind gift of K. Leenhouts, BioMade, Groningen, The Netherlands). The strain was grown at $30{ }^{\circ} \mathrm{C}$ in $\mathrm{M} 17$ glucose medium containing $10 \mathrm{ng}$ nisin $\mathrm{ml}^{-1}$ to induce the production of $\mathrm{c}-\mathrm{Myc}-\mathrm{PA}$ fusion protein. The supernatant was recovered at the end of the exponential growth phase and filtered through a $0.22 \mu \mathrm{m}$ pore-size filter. Peptidoglycan $(150 \mu \mathrm{g})$ extracted from the different $L$. lactis strains was resuspended in $1 \mathrm{ml}$ of culture supernatant containing c-Myc-PA diluted 10,20 or 50 times in culture supernatant of L. lactis MG1363acmA $\Delta 1$. The suspensions were incubated for $1 \mathrm{~h}$ at room temperature under rotational shaking and centrifuged for $30 \mathrm{~min}$ at $35000 \mathrm{~g}$, and the pellet was washed twice with PBS. Then, the pellet was resuspended in $40 \mu \mathrm{l}$ denaturing buffer $(50 \mathrm{mM}$ Tris/ $\mathrm{HCl}, \mathrm{pH} 6.8$, $2 \%$ SDS, $10 \%$, v/v, glycerol, $0.1 \%$ Bromophenol Blue and $100 \mathrm{mM}$ DTT), boiled for $3 \mathrm{~min}$ and centrifuged for $10 \mathrm{~min}$ at $35000 \mathrm{~g}$ before SDS-PAGE. Supernatant $(10 \mu \mathrm{l})$ was analysed by SDS-PAGE with a $12.5 \%$ polyacrylamide gel. c-Myc-PA protein bound to peptidoglycan was detected by Western blot hybridization after transfer of the proteins onto a nitrocellulose membrane (Bio-Rad), as described elsewhere (Towbin et al., 1979). Membranes were incubated successively with monoclonal anti-c-Myc antibody (Clontech) and rabbit anti-mouse antibodies coupled to horseradish peroxidase (Amersham), and revealed by chemiluminescence with a Western Lightning kit (Perkin Elmer) according to the manufacturer's instructions. The resulting light was detected on Hyperfilm ECL (Amersham). The film was scanned with a Duoscan T1200 scanner (Agfa-Gevaert) and quantitative analysis of revealed bands was performed with ImageJ software (http://rsb.info.nih.gov/ij/). The relative amount of $\mathrm{c}-\mathrm{Myc}-\mathrm{PA}$ protein in the different samples was standardized against the amount of c-Myc-PA contained in $5 \mu$ L. lactis PA1001(pPA3) supernatant, which was run on each gel.

\section{RESULTS}

\section{Analysis of $L$. lactis pgdA sequence}

The gene $p g d A(x y n D)$ encodes a 372-residue protein with a putative N-terminal signal peptide, predicted by Psort software (http://psort.hgc.jp) to be uncleavable. The PgdA amino acid sequence exhibits $31 \%$ identity to the $S$. pneumoniae peptidoglycan GlcNAc deacetylase sequence (Vollmer \& Tomasz, 2000). In addition, L. lactis PgdA contains a conserved zinc-binding triad (Asp-184, His-234, His-238) identified in the active site of its $S$. pneumoniae homologue (Blair et al., 2005). Northern blot analysis indicated that the $p g d A$ gene is transcribed during the exponential growth phase in L. lactis IL1403 (data not shown). The transcript size $(1.3 \mathrm{~kb})$ indicated a monocistronic organization of the gene, in agreement with the detection of two putative rho-independent transcription terminators upstream and downstream of $p g d A$.

\section{L. lactis pgdA encodes a peptidoglycan GIcNAc deacetylase}

To investigate the role of $p g d A$, the gene was inactivated in L. lactis subsp. lactis strain IL6288. This strain is a derivative of IL1403 obtained after curing the six identified prophages (Chopin et al., 2001) from the chromosome (A. Aucouturier and M.-C. Chopin, personal communication). Inactivation of $p g d A$ was obtained by SCO integration of the non-replicative vector pJIM2242 containing a 597 bp internal $p g d A$ fragment.

The peptidoglycan structure of the IL6288 $p g d A$ mutant (TIL926) was analysed and compared with that of the parental strain IL6288. After peptidoglycan digestion with mutanolysin, a muramidase, the resulting muropeptides were separated by HPLC (Fig. 1). Four peaks (indicated by arrows) present in the IL6288 muropeptide profile (Fig. 1a) were absent from the TIL926 muropeptide profile (Fig. 1b). These peaks (A, B, E, F) (Fig. 1c) were restored in the muropeptide profile of the complemented strain TIL926(pJIMpgdA), obtained by cloning pgdA into the multicopy plasmid vector pJIM2246. Also, three other minor peaks $(\mathrm{C}, \mathrm{D}, \mathrm{G})$ appeared in the complemented mutant (Fig. 1c). Peaks A-G were analysed by MALDITOF MS. Their measured $\mathrm{m} / z$ values exhibited a $42 \mathrm{Da}$ mass defect compared to the masses expected for the molecular sodiated ions corresponding to L. lactis fully acetylated muropeptides (Table 2). This 42 Da mass defect corresponds to the loss of one acetyl group. Thus, all the peaks A-G contained deacetylated muropeptides. From these results, we conclude that $p g d A$ encodes a peptidoglycan deacetylase. The percentage of deacetylated muropeptides was calculated to be $9.8 \%$ in the parental strain IL6288 at the beginning of exponential growth, whereas it was $15.8 \%$ in the complemented strain TIL926(pJIMpgdA). These data indicate that $p g d A$ is overexpressed in TIL926(pJIMpgdA), most probably because $p g d A$ was cloned on a high-copy-number plasmid.

Peak A, containing the deacetylated monomer disaccharide tripeptide with Asn side chain $(\mathrm{m} / z$ 920.45), was subjected to MALDI-PSD analysis, which allowed the analysis of muropeptide fragment ions (Fig. 2). A fragment with $\mathrm{m} / \mathrm{z}$ of 760.6 corresponded to the loss of one glucosamine. Several other fragments resulted from the loss of one glucosamine $(\mathrm{m} / \mathrm{z} 161)$, whereas no fragment corresponding to the loss of one GlcNAc $(\mathrm{m} / z$ 203) was observed. These results indicate that muropeptide A contains glucosamine instead of GlcNAc, and that deacetylation occurs on GlcNAc. Thus, the L. lactis pgdA gene encodes a peptidoglycan GlcNAc deacetylase. 


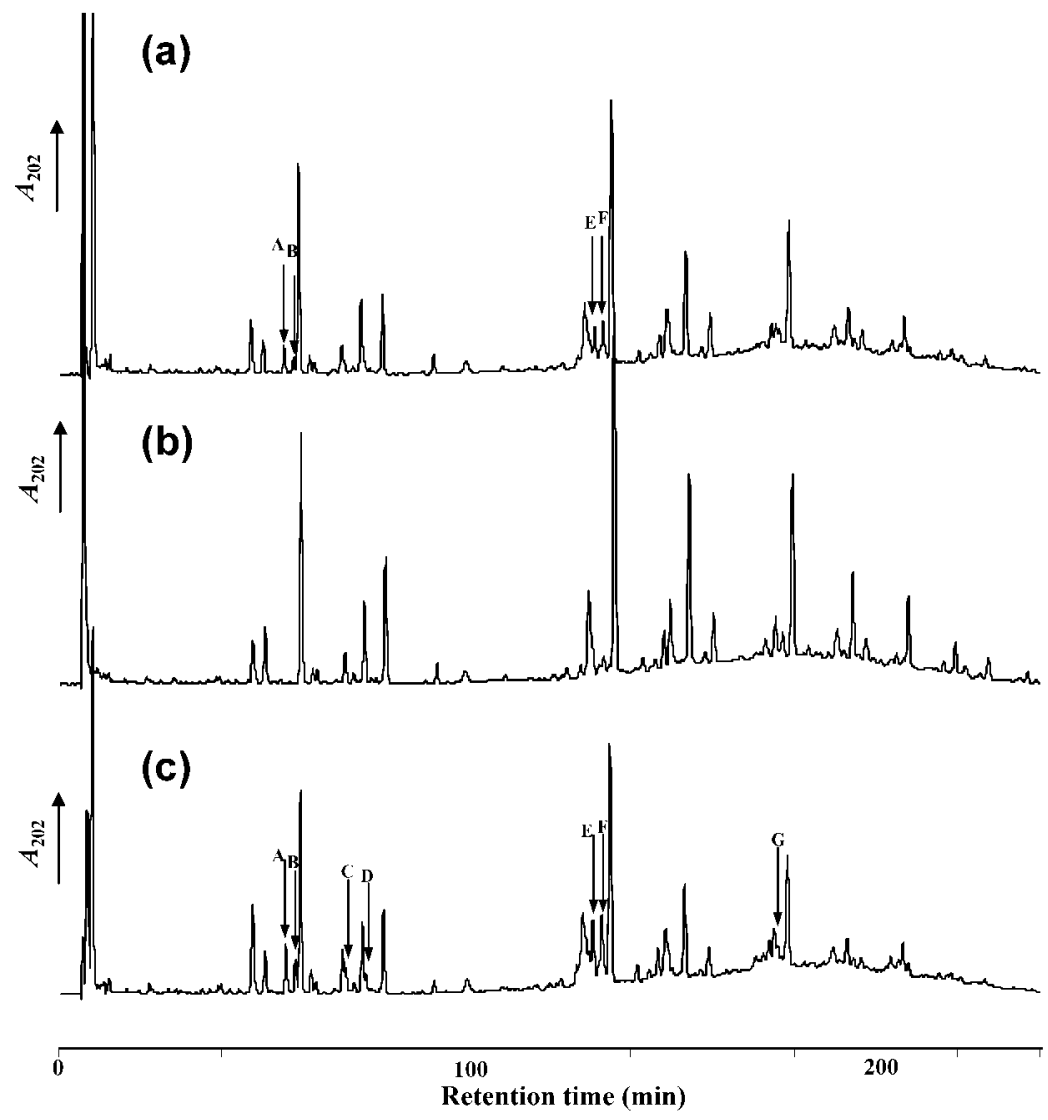

Fig. 1. Reversed-phase HPLC separation of muropeptides from L. lactis IL6288 (a), IL6288 pgdA mutant (TIL926) (b) and complemented mutant TIL926(pJIMpgdA) (c). Arrows indicate peaks which contain deacetylated muropeptides.

\section{Influence of peptidoglycan de- $\mathrm{N}$-acetylation on lysozyme sensitivity}

We tested the lysozyme sensitivity of IL6288 and its derivative strains by plating cultures of each strain on M17 agar plates containing lysozyme concentrations from 0 to $3 \mathrm{mg} \mathrm{ml}^{-1}$. The parental strain IL6288 was resistant to $1 \mathrm{mg}$ lysozyme $\mathrm{ml}^{-1}$, whereas the $p g d A$-negative mutant TIL926 was sensitive to $1 \mathrm{mg} \mathrm{ml}^{-1}$. In contrast, the complemented strain TIL926(pJIMpgdA) with a higher

Table 2. Structures, molecular masses and quantities of deacetylated muropeptides

\begin{tabular}{|c|c|c|c|c|c|c|}
\hline \multirow[t]{2}{*}{ Peak $^{*}$} & \multirow[t]{2}{*}{ Observed $\mathrm{m} / \mathrm{z}$} & \multirow{2}{*}{$\begin{array}{l}\text { Calculated } \\
{[\mathrm{M}+\mathrm{Na}]^{+} \dagger}\end{array}$} & \multirow[t]{2}{*}{$\Delta \mathbf{m}$} & \multirow[t]{2}{*}{ Identification $\ddagger$} & \multicolumn{2}{|c|}{ Percentage $\$$} \\
\hline & & & & & IL6288 & TI1926(pJIM $p g d A)$ \\
\hline B & 921.35 & 963.41 & -42.06 & Tri-D (deAc) & 0.7 & 1.4 \\
\hline $\mathrm{C}$ & 991.49 & 1033.47 & -41.98 & Tetra-N (deAc) & 0.4 & 1.0 \\
\hline $\mathrm{F}$ & 1912.90 & 1954.90 & -42.00 & Tri-N-Tetra-N (deAc) & 3.1 & 4.7 \\
\hline \multirow[t]{2}{*}{ G } & 2905.22 & 2947.36 & -42.14 & Tri-N-Tetra-N-Tetra-N (deAc) & 1.4 & 1.8 \\
\hline & & & & Total & 9.8 & 15.8 \\
\hline
\end{tabular}

${ }^{*}$ Peak identification refers to the chromatograms in Fig. 1 .

$\dagger$ Calculated $[\mathrm{M}+\mathrm{Na}]^{+}$corresponds to the non-deacetylated muropeptides from L. lactis. Sodiated molecular ions were the most abundant ones in MALDI-TOF mass spectra for all muropeptides.

$\ddagger$ Tri, disaccharide tripeptide (L-Ala-D-iGln-L-Lys); Tetra, disaccharide tetrapeptide (L-Ala-D-iGln-L-Lys-D-Ala); Penta, disaccharide pentapeptide (L-Ala-D-iGln-L-Lys-D-Ala-D-Ala). Disaccharide=GlcNAc-MurNAc; D, Asp; N, Asn; deAc, deacetylation; iGln, isoGln.

\$The percentage for each peak was calculated as the ratio of its area over the sum of all the peak areas. 
(a)

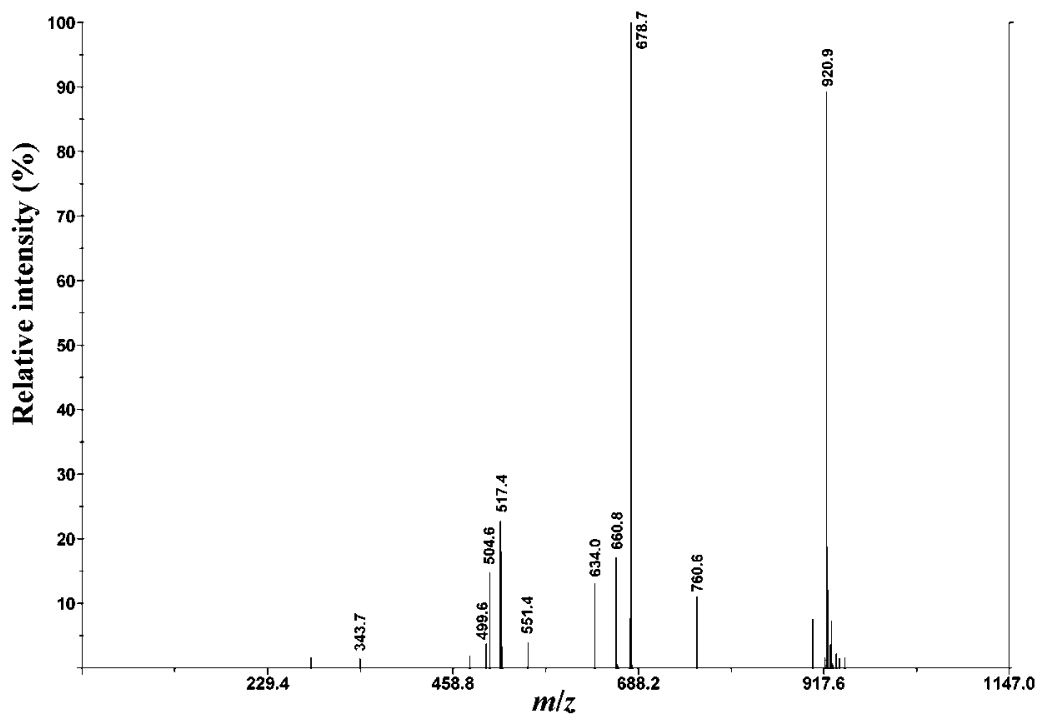

(b)

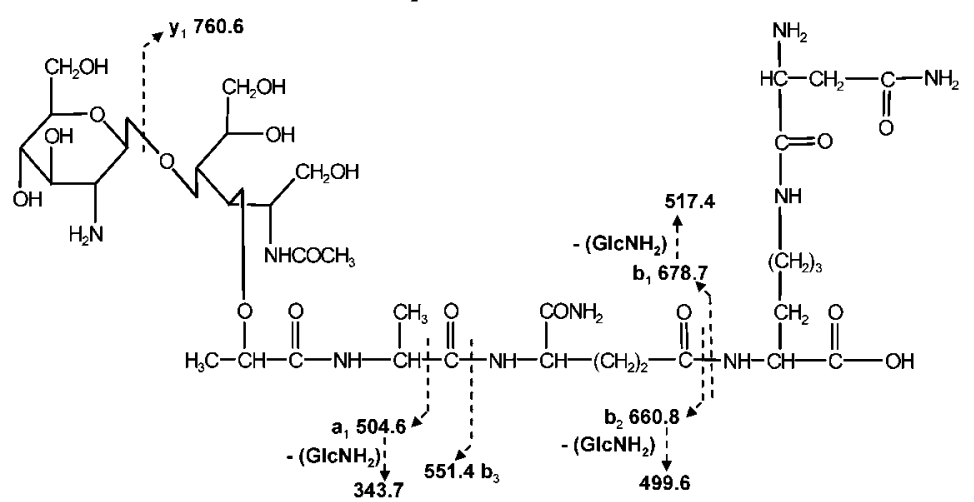

Fig. 2. MALDI-PSD analysis of reduced muropeptide A purified by reversed-phase HPLC (see Fig. 1). The $[\mathrm{M}+\mathrm{Na}]^{+}$parental ion $(\mathrm{m} / \mathrm{z}$ 920.9) was selected for PSD analysis. (a) MALDI-PSD TOF mass spectrum of sodiated ions corresponding to fragments that issued from muropeptide $A$; (b) chemical structure determined for muropeptide $A$ and fragmention pattern. The dashed arrows indicate $\mathrm{N}$-terminal fragment ions ( $\mathrm{a}$ and $\mathrm{b}$ ions) and a C-terminal fragment ion (ion y) identified on the spectrum. Several fragments result from the loss of one glucosamine $(\mathrm{m} / \mathrm{z} 161)$. degree of peptidoglycan deacetylation showed increased resistance to lysozyme and grew in the presence of $2 \mathrm{mg}$ lysozyme $\mathrm{ml}^{-1}$, while the parent strain did not.

\section{Influence of peptidoglycan de- $\mathrm{N}$-acetylation on cell separation and autolysis}

The growth rates of the $p g d A$-negative mutant and the wild-type strain were identical in M17 glucose medium, and the growth rates of the complemented strain TIL926(pJIMpgdA) and the control TIL926(pJIM) strain were identical in M17 glucose medium containing chloramphenicol. These results indicate that inactivation or overexpression of $p g d A$ has no impact on bacterial growth rate. Microscopic observation of the different strains revealed that strain TIL926(pJIMpgdA) forms long chains of cells (Fig. 3c), unlike the parental strain IL6288 (Fig. 3a) and the pgdA-negative mutant TIL926 (Fig. 3b). The chain length for TIL926(pJIMpgdA) (15-20 cells) was shorter than that obtained with the MG1363 acmA mutant (50-100 cells per chain). The defect of cell separation in TIL926(pJIMpgdA) may result from the resistance of deacetylated peptidoglycan to endogenous autolysins.

Autolysis of the different strains after they had reached stationary phase was followed over 6 days. During this period, TIL926(pJIMpgdA) culture did not exhibit autolysis, as measured by the decrease in $\mathrm{OD}_{600}$, and behaved like the MG1363acmA mutant (data not shown). No difference in the rate and extent of autolysis was found among the wild-type IL6288 strain, the pgdA-negative mutant and the control strain TIL926(pJIM). For the three strains, the decrease in $\mathrm{OD}_{600}$ reached $10 \%$ after 4 days, and then remained stable.

Autolysis was also compared after transfer of bacterial cells to $50 \mathrm{mM}$ potassium phosphate buffer, $\mathrm{pH} 7.0$, containing $0.05 \%$ Triton X-100. As shown in Fig. 4, the pgdA mutant TIL926 exhibited a similar initial autolysis rate to that of 
(a)

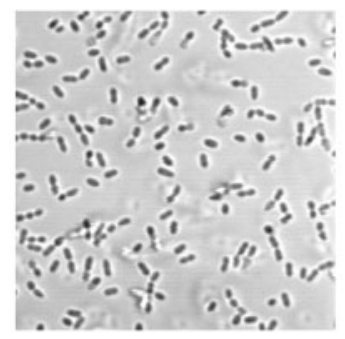

(b)

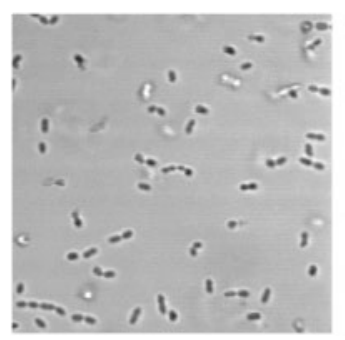

(c)

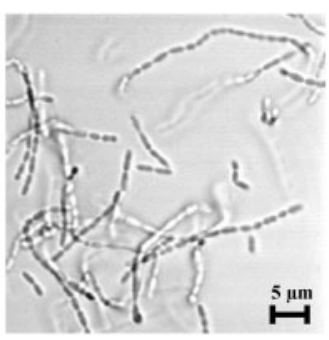

Fig. 3. Phase-contrast microscopy of L. lactis IL6288 (a), the TIL926 mutant (b) and the complemented TIL926(pJIMpgdA) mutant (c). the parental IL6288 strain, but a higher final autolysis level. In contrast, strain TIL926(pJIMpgdA), with a higher degree of peptidoglycan de- $\mathrm{N}$-acetylation, showed a reduced rate of autolysis compared to IL6288. The control strain TIL926(pJIM) exhibited an autolysis rate and a final extent of autolysis identical to those of TIL926 without plasmid (data not shown).

\section{Influence of GIcNAc deacetylation on peptidoglycan susceptibility to AcmA autolysin}

Since AcmA autolysin is the major enzyme involved in $L$. lactis autolysis and is involved in cell separation after cell division (Buist et al., 1995), we tested the activity of AcmA in vitro on peptidoglycan substrates with different degrees of de- $\mathrm{N}$-acetylation.

AcmA without its putative signal sequence and with a Cterminal hexa-His-tag was produced in E. coli

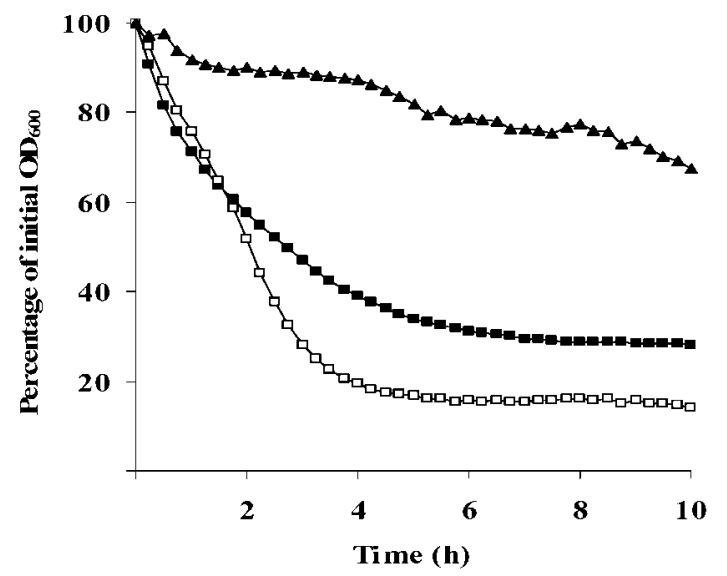

Fig. 4. Autolysis of L. lactis IL6288 and its derivative mutants in buffer containing Triton $\mathrm{X}-100$. Cells were grown in M17 glucose medium to $\mathrm{OD}_{600} 0.8$, and washed and resuspended at $\mathrm{OD}_{600} 1$ in $50 \mathrm{mM}$ potassium phosphate, $\mathrm{pH} 7.0$, containing $0.05 \%$ Triton $\mathrm{X}-100$ at $30{ }^{\circ} \mathrm{C}$. Lysis of IL6288 (ם), pgdA mutant TIL926 ( $\square$ ) and TIL926(pJIMpgdA) ( $\mathbf{\Delta}$ ) was monitored by measuring the decrease in $\mathrm{OD}_{600}$ of the cell suspensions. Optical density values are the mean of three experiments made with one culture, and SD values were close to zero. The results are representative of three independent experiments.
BL21(DE3)pLysE under control of the T7 promoter. After induction with IPTG, a low amount of recombinant AcmA-His was obtained (data not shown). The protein was purified by affinity chromatography on a His-Trap column. The major protein detected at $45 \mathrm{kDa}$ in the purified fraction analysed by SDS-PAGE corresponded to AcmA-His (Fig. 5a, lane 1), as confirmed by PMF analysis. In addition, its activity could be detected by zymogram assay with M. luteus cells as substrate (Fig. 5b, lane 1). Three minor contaminant proteins were co-purified, and were identified by PMF as E. coli proteins not related to PGHs (Fig. 5a, lane 1). These contaminant proteins did not exhibit peptidoglycan-hydrolysing activity as checked by zymogram assay (Fig. 5b, lane 1). Thus, we used the partially purified AcmA-His for activity tests.

AcmA activity was first tested on autoclaved cells obtained from the three strains which exhibited different degrees of peptidoglycan de- $N$-acetylation: IL6288 (9.8\%), pgdAnegative mutant TIL926 (0\%) and complemented strain TIL926(pJIMpgdA) (15.8\%). Activity was measured by the optical density decrease of the cell suspensions. As shown
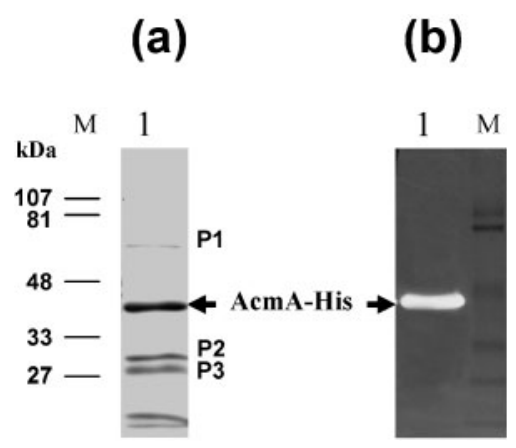

Fig. 5. SDS-PAGE (a) and zymogram (b) analysis of purified AcmA-His. Proteins were separated on $10 \%$ polyacrylamide gels. (a) Protein bands were stained with Coomassie Blue; (b) the gel contained $0.2 \%(w / v)$ M. luteus cells. Lane 1 contained His-Trap column eluate (300 ng protein). P1, P2 and P3 designate proteins which were co-purified with AcmA-His. They were identified by PMF analysis as E. coli proteins: P1, UDP-D-glucuronate dehydrogenase (YfbG); P2, 50 S ribosomal protein L2; P3, FKBP-type peptidyl-prolyl cis-trans isomerase (SlyD). M, molecular mass markers. 
in Fig. 6a, the highest activity was found towards cells originating from the negative mutant TIL926 with fully acetylated peptidoglycan. AcmA activity towards cells from TIL926(pJIMpgdA) with the highest degree of de- $\mathrm{N}$-acetylation was lower than towards parental strain IL6288 cells. These results indicate that de- $N$-acetylation of peptidoglycan reduces its susceptibility to the autolysin AcmA.

We further confirmed this result by testing AcmA-His activity in a zymogram assay with purified peptidoglycan as substrate included into polyacrylamide gels. As shown in Fig. 6b, when the same amounts of AcmA-His were loaded on the gels, the activity band detected on peptidoglycan from the $p g d A$-negative mutant was more intense than that on peptidoglycan from TIL926(pJIMpgdA), confirming the lower activity of AcmA towards de- $N$-acetylated peptidoglycan compared to fully acetylated peptidoglycan.

\section{Influence of GIcNAc deacetylation on AcmA binding to $L$. lactis peptidoglycan}

AcmA produced by L. lactis cells is found both attached to the cells and in the culture supernatant (Buist et al., 1995). It has to bind the peptidoglycan of the cell wall through its C-terminal LysM-containing domain to be able to lyse the cells (Steen et al., 2003). We investigated whether peptidoglycan deacetylation influences substrate binding of AcmA.

First, using the zymogram technique, we compared the amount of AcmA bound to the cells and the amount present in culture supernatant for IL6288 and its derivative mutants. No difference was found in the intensity of the AcmA activity band on micrococci used as substrate between the $p g d A$ mutant and wild-type IL6288 or the overexpressing strain (data not shown). Breakdown products of AcmA, which retained activity in the zymogram (Poquet et al., 2000; Steen et al., 2003), were barely detected in IL6288 and were present in similar amounts in its derivative mutants, indicating no difference in AcmA degradation among the tested strains.

Second, we examined whether peptidoglycan de- $\mathrm{N}$-acetylation affects AcmA binding to purified peptidoglycan. AcmA consists of two domains: an active site domain and a C-terminal region containing three highly homologous repeats of 45 aa, named LysM domains, which bind peptidoglycan (Steen et al., 2003). To study the binding of AcmA to peptidoglycan with different degrees of de- $\mathrm{N}$ acetylation, we used a fusion protein consisting of the Cterminal peptidoglycan-binding domain of AcmA fused to a c-Myc epitope (c-Myc-PA) at its $\mathrm{N}$ terminus (Bosma et al., 2006). Peptidoglycan extracted from IL6288, $p g d A$ mutant TIL926 and pgdA-overexpressing strain TIL926(pJIMpgdA) was incubated with different concentrations of c-Myc-PA. The amount of fusion protein bound to each peptidoglycan was analysed by Western blotting with specific anti-c-Myc antibody (Fig. 7). A band with the expected molecular mass $(28 \mathrm{kDa})$ was detected. Quantitative analysis of the immunodetected band in each sample (data not shown) indicated that equal amounts of c-Myc-PA bound to peptidoglycan from the wild-type, $p g d A$ mutant and $p g d A$-overexpressing strains, with different degrees of deacetylation. These results indicate that GlcNAc deacetylation does not modify AcmA binding to peptidoglycan in strain TIL926(pJIMpgdA), but most probably affects the efficiency of peptidoglycan chain cleavage by AcmA.

\section{DISCUSSION}

The aim of the study was to evaluate the impact of peptidoglycan structural modification on L. lactis autolysis. We have identified a peptidoglycan deacetylase (PgdA) in $L$. lactis which deacetylates GlcNAc residues. We have shown that the increase of $\mathrm{N}$-unsubstituted glucosamine residues in peptidoglycan results in decreased autolysis of (a)

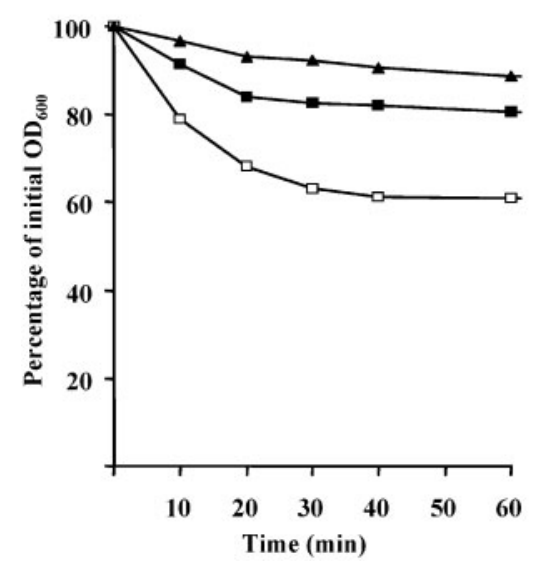

(b)

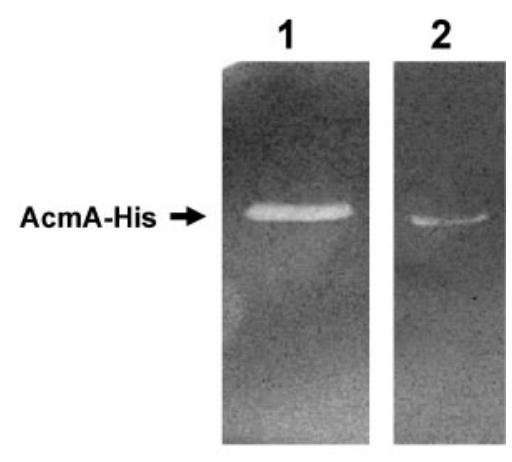

Fig. 6. (a) Activity of AcmA-His towards autoclaved cells with different degrees of de$\mathrm{N}$-acetylation. Purified AcmA-His was incubated with autoclaved cells prepared from $L$.

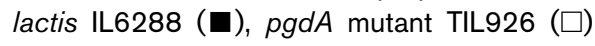
and TIL926(pJIMpgdA) ( $\mathbf{\Delta})$, and the decrease in $\mathrm{OD}_{600}$ of the cell suspensions was monitored. The results shown are representative of three independent experiments in which similar results were obtained. (b) Zymogram analysis of AcmA-His activity towards peptidoglycan substrates included in the gels. Lanes: 1, peptidoglycan from pgdA mutant TIL926; 2, peptidoglycan from TIL926(pJIMpgdA). Purified AcmA-His ( $450 \mathrm{ng}$ ) was loaded on each gel. 


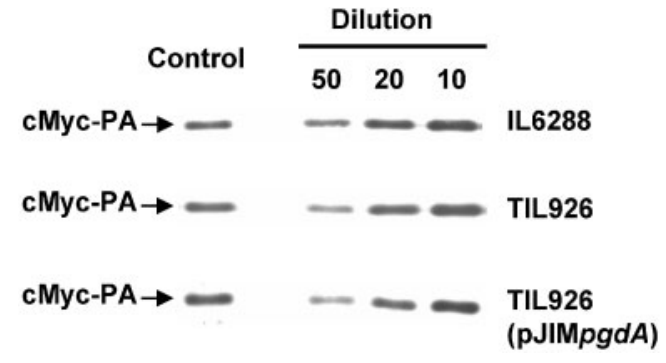

Fig. 7. Western blot analysis of the binding of c-Myc-PA fusion protein to purified peptidoglycan with different degrees of de- $N$ acetylation. Peptidoglycan $(150 \mu \mathrm{g})$ extracted from IL6288, pgdA mutant TIL926 and TIL926(pJIMpgdA) was incubated with culture supernatant of PA1001(pPA3) containing c-Myc-PA protein diluted 10,20 or 50 times in MG1363acmA $\Delta 1$ culture supernatant. Bound c-Myc-PA protein was detected with specific anti-cMyc antibody. A control sample [5 $\mu$ l PA1001(pPA3) supernatant] was included in each experiment and was used as a standard for the quantitative analysis of the immunodetected bands.

L. lactis cells due to reduced susceptibility of the modified peptidoglycan to the hydrolytic activity of the L. lactis major autolysin AcmA.

GlcNAc deacetylation of peptidoglycan negatively influences the hydrolytic activity of AcmA, which is a glucosaminidase, able to hydrolyse $\beta$-1,4-glycosidic bonds between GlcNAc and MurNAc. Deacetylation of peptidoglycan aminosugars has previously been shown to affect the hydrolytic activity of PGHs with different specificities, either positively or negatively. For example, de- $\mathrm{N}$-acetylation of peptidoglycan GlcNAc confers resistance to lysozyme, an exogenous muramidase, upon several bacterial species, such as S. pneumoniae (Vollmer \& Tomasz, 2000), Bacillus cereus (Hayashi et al., 1973), Listeria monocytogenes (Boneca et al., 2007) and L. lactis (this study). In contrast, a muramidase purified from Clostridium acetobutylicum has been found to act on non-acetylated peptidoglycan alone (Croux et al., 1992). Also, the S. pneumoniae LytA amidase has higher activity towards non-modified S. pneumoniae peptidoglycan with a high degree of deacetylation than towards chemically acetylated peptidoglycan (Vollmer \& Tomasz, 2000). In $B$. cereus, glycosidases have been identified with a preference for either peptidoglycan with $\mathrm{N}$-unsubstituted glucosamine or peptidoglycan with acetylated aminosugars (Kawagishi et al., 1980).

AcmA consists of two domains: an active site domain and a C-terminal region containing three highly homologous repeats of 45 aa, named LysM domains, which bind peptidoglycan. We found that equal amounts of AcmA C-terminal domain bound to peptidoglycan with the different degrees of de- $N$-acetylation tested $(15.8 \%$ in the $p g d A$-overexpressing strain vs $0 \%$ in the $p g d A$-negative mutant). Thus, the reduced autolysis of $L$. lactis cells with an increased degree of peptidoglycan deacetylation does not seem to result from reduced AcmA binding to the cell wall peptidoglycan substrate. The decreased autolysis therefore probably results from reduced efficiency of cleavage by AcmA of $\mathrm{GlcNH}_{2}-\mathrm{MurNAc}$ bonds compared to GlcNAc-MurNAc bonds.

The degree of peptidoglycan GlcNAc deacetylation in $L$. lactis strain IL6288 $(9.8 \%)$ was low compared to that observed in S. pneumoniae (over $80 \%$ in strain R36A; Vollmer \& Tomasz, 2000), B. cereus (40-100\%; Hayashi et al., 1973) and L. monocytogenes (50\%; Boneca et al., 2007). However, this appears to be sufficient to modulate the sensitivity of L. lactis cells to lysozyme and to AcmA autolysin.

The autolysis of $L$. lactis is of special interest with respect to its use as a starter in dairy fermentations. It has been shown that bacterial autolysis during cheese ripening enhances the contribution of intracellular enzymes to cheese flavour formation (Lortal \& Chapot-Chartier, 2005). For another proposed application of L. lactis, i.e. its use as a delivery vehicle for antigens and therapeutic molecules in the digestive tract of humans and animals (Nouaille et al., 2003), autolysis is also a critical parameter to consider for optimal delivery of molecules (Grangette et al., 2004). The autolytic properties of $L$. lactis have been observed to be strain-dependent (Lortal \& Chapot-Chartier, 2005). In this study, we observed that the degree of peptidoglycan deacetylation influences autolytic properties. Variation in the degree of peptidoglycan deacetylation could be one of the factors that contributes to the variability of autolytic properties among natural strains.

Since PGHs are potentially lethal enzymes for the cells that produce them, their activities are thought to be regulated at the post-translational level (Shockman \& Höltje, 1994; Smith et al., 2000). This regulation could involve different factors. With respect to AcmA, previous studies have already revealed two mechanisms involved in the control of its activity in L. lactis cells. First, AcmA is found to bind to peptidoglycan through its LysM domains at specific loci in the cell wall, around the poles and septum of the cells, whereas AcmA binding on the whole cell surface is hindered by some cell wall constituents, most probably by lipoteichoic acids (Steen et al., 2003). Second, it has been shown that a decrease of the level of D-Ala substitution on lipoteichoic acids leads to increased autolysis through an indirect effect that results from the decreased degradation of AcmA by the extracellular housekeeping protease HtrA (Steen et al., 2005b). The degree of peptidoglycan de- $\mathrm{N}$-acetylation could constitute a third mechanism of control of AcmA in cells by decreasing the susceptibility of peptidoglycan to AcmA.

\section{ACKNOWLEDGEMENTS}

We are very grateful to M.-C. Chopin, INRA, Jouy-en-Josas, for the gift of strain IL6288, K. Leenhouts, BioMade, Groningen, for strain PA1001(pPA3) and G. Buist, University of Groningen, for the 
MG1363acmA mutant. We thank Romain Briandet for his valuable help in microscopy experiments, Céline Henry (PAPSS, Unité de Biochimie Bactérienne, INRA, Jouy-en-Josas) for helpful advice in PMF analysis, Colette Besset for Northern blotting and Michèle Nardi for helpful technical advice. We thank V. Monnet, M. Yvon and S. Kulakauskas for critical reading of the manuscript.

\section{REFERENCES}

Atrih, A., Bacher, G., Allmaier, G., Williamson, M. P. \& Foster, S. J. (1999). Analysis of peptidoglycan structure from vegetative cells of Bacillus subtilis 168 and role of PBP 5 in peptidoglycan maturation. $J$ Bacteriol 181, 3956-3966.

Blair, D. E., Schuttelkopf, A. W., MacRae, J. I. \& van Aalten, D. M. (2005). Structure and metal-dependent mechanism of peptidoglycan deacetylase, a streptococcal virulence factor. Proc Natl Acad Sci U S A 102, 15429-15434.

Boneca, I. G., Dussurget, O., Cabanes, D., Nahori, M. A., Sousa, S., Lecuit, M., Psylinakis, E., Bouriotis, V., Hugot, J. P. \& other authors (2007). A critical role for peptidoglycan $N$-deacetylation in Listeria evasion from the host innate immune system. Proc Natl Acad Sci U S A 104, 997-1002.

Bosma, T., Kanninga, R., Neef, J., Audouy, S. A., van Roosmalen, M. L., Steen, A., Buist, G., Kok, J., Kuipers, O. P. \& other authors (2006). Novel surface display system for proteins on nongenetically modified Gram-positive bacteria. Appl Environ Microbiol 72, 880-889.

Buist, G., Kok, J., Leenhouts, K. J., Dabrowska, M., Venema, G. \& Haandrikman, A. J. (1995). Molecular cloning and nucleotide sequence of the gene encoding the major peptidoglycan hydrolase of Lactococcus lactis, a muramidase needed for cell separation. J Bacteriol 177, 1554-1563.

Buist, G., Venema, G. \& Kok, J. (1998). Autolysis of Lactococcus lactis is influenced by proteolysis. J Bacteriol 180, 5947-5953.

Calamita, H. G., Ehringer, W. D., Koch, A. L. \& Doyle, R. J. (2001). Evidence that the cell wall of Bacillus subtilis is protonated during respiration. Proc Natl Acad Sci U S A 98, 15260-15263.

Chaurand, P., Luetzenkirchen, F. \& Spengler, B. (1999). Peptide and protein identification by matrix-assisted laser desorption ionization (MALDI) and MALDI-post-source decay time-of-flight mass spectrometry. J Am Soc Mass Spectrom 10, 91-103.

Chich, J. F., Rigolet, P., Nardi, M., Gripon, J. C., Ribadeau-Dumas, B. \& Brunie, S. (1995). Purification, crystallization, and preliminary Xray analysis of PepX, an X-prolyl dipeptidyl aminopeptidase from Lactococcus lactis. Proteins 23, 278-281.

Chopin, A., Chopin, M. C., Moillo-Batt, A. \& Langella, P. (1984). Two plasmid-determined restriction and modification systems in Streptococcus lactis. Plasmid 11, 260-263.

Chopin, A., Bolotin, A., Sorokin, A., Ehrlich, S. D. \& Chopin, M. (2001). Analysis of six prophages in Lactococcus lactis IL1403: different genetic structure of temperate and virulent phage populations. Nucleic Acids Res 29, 644-651.

Cornett, J. B. \& Shockman, G. D. (1978). Cellular lysis of Streptococcus faecalis induced with Triton X-100. J Bacteriol 135, 153-160.

Courtin, P., Miranda, G., Guillot, A., Wessner, F., Mezange, C., Domakova, E., Kulakauskas, S. \& Chapot-Chartier, M. P. (2006). Peptidoglycan structure analysis of Lactococcus lactis reveals the presence of an L,D-carboxypeptidase involved in peptidoglycan maturation. J Bacteriol 188, 5293-5298.

Coutinho, P. M. \& Henrissat, B. (1999). Carbohydrate-active enzymes: an integrated database approach. In Recent Advances in Carbohydrate
Bioengineering, pp. 3-12. Edited by H. J. Gilbert, G. Davies, B. Henrissat \& B. Svensson. Cambridge: The Royal Society of Chemistry.

Croux, C., Canard, B., Goma, G. \& Soucaille, P. (1992). Purification and characterization of an extracellular muramidase of Clostridium acetobutylicum ATCC 824 that acts on non- $\mathrm{N}$-acetylated peptidoglycan. Appl Environ Microbiol 58, 1075-1081.

Delcour, J., Ferain, T., Deghorain, M., Palumbo, E. \& Hols, P. (1999). The biosynthesis and functionality of the cell-wall of lactic acid bacteria. Antonie Van Leeuwenhoek 76, 159-184.

Grangette, C., Muller-Alouf, H., Hols, P., Goudercourt, D., Delcour, J., Turneer, M. \& Mercenier, A. (2004). Enhanced mucosal delivery of antigen with cell wall mutants of lactic acid bacteria. Infect Immun 72, 2731-2737.

Guedon, E., Serror, P., Ehrlich, S. D., Renault, P. \& Delorme, C. (2001). Pleiotropic transcriptional repressor CodY senses the intracellular pool of branched-chain amino acids in Lactococcus lactis. Mol Microbiol 40, 1227-1239.

Guillot, A., Gitton, C., Anglade, P. \& Mistou, M. Y. (2003). Proteomic analysis of Lactococcus lactis, a lactic acid bacterium. Proteomics 3, 337-354.

Hayashi, H., Araki, Y. \& Ito, E. (1973). Occurrence of glucosamine residues with free amino groups in cell wall peptidoglycan from bacilli as a factor responsible for resistance to lysozyme. J Bacteriol 113, 592-598.

Holo, H. \& Nes, I. F. (1989). High-frequency transformation by electroporation of Lactococcus lactis subsp. cremoris grown with glycine in osmotically stabilized media. Appl Environ Microbiol 55, 3119-3123.

Huard, C., Miranda, G., Wessner, F., Bolotin, A., Hansen, J., Foster, S. J. \& Chapot-Chartier, M. P. (2003). Characterization of AcmB, an $\mathrm{N}$-acetylglucosaminidase autolysin from Lactococcus lactis. Microbiology 149, 695-705.

Huard, C., Miranda, G., Redko, Y., Wessner, F., Foster, S. J. \& Chapot-Chartier, M. P. (2004). Analysis of the peptidoglycan hydrolase complement of Lactococcus lactis: identification of a third $\mathrm{N}$ acetylglucosaminidase, AcmC. Appl Environ Microbiol 70, 3493-3499.

Kawagishi, S., Araki, Y. \& Ito, E. (1980). Bacillus cereus autolytic endoglucosaminidase active on cell wall peptidoglycan with $N$ unsubstituted glucosamine residues. J Bacteriol 141, 137-143.

Kemper, M. A., Urrutia, M. M., Beveridge, T. J., Koch, A. L. \& Doyle, R. J. (1993). Proton motive force may regulate cell wall-associated enzymes of Bacillus subtilis. J Bacteriol 175, 5690-5696.

Lazarevic, V., Margot, P., Soldo, B. \& Karamata, D. (1992). Sequencing and analysis of the Bacillus subtilis lytRABC divergon: a regulatory unit encompassing the structural genes of the $\mathrm{N}$ acetylmuramoyl-L-alanine amidase and its modifier. J Gen Microbiol 138, 1949-1961.

Lortal, S. \& Chapot-Chartier, M. P. (2005). Role, mechanisms and control of lactic acid bacteria lysis in cheese. Int Dairy J 15, 857-871.

Nouaille, S., Ribeiro, L. A., Miyoshi, A., Pontes, D., Le Loir, Y., Oliveira, S. C., Langella, P. \& Azevedo, V. (2003). Heterologous protein production and delivery systems for Lactococcus lactis. Genet Mol Res 2, 102-111.

Palumbo, E., Deghorain, M., Cocconcelli, P. S., Kleerebezem, M., Geyer, A., Hartung, T., Morath, S. \& Hols, P. (2006). D-Alanyl ester depletion of teichoic acids in Lactobacillus plantarum results in a major modification of lipoteichoic acid composition and cell wall perforations at the septum mediated by the Acm2 autolysin. $J$ Bacteriol 188, 3709-3715.

Pfeffer, J. M., Strating, H., Weadge, J. T. \& Clarke, A. J. (2006). Peptidoglycan $\mathrm{O}$ - acetylation and autolysin profile of Enterococcus faecalis in the viable but nonculturable state. J Bacteriol $\mathbf{1 8 8}$, 902-908. 
Poquet, I., Saint, V., Seznec, E., Simoes, N., Bolotin, A. \& Gruss, A. (2000). HtrA is the unique surface housekeeping protease in Lactococcus lactis and is required for natural protein processing. Mol Microbiol 35, 1042-1051.

Psylinakis, E., Boneca, I. G., Mavromatis, K., Deli, A., Hayhurst, E., Foster, S. J., Varum, K. M. \& Bouriotis, V. (2005). Peptidoglycan $N$ acetylglucosamine deacetylases from Bacillus cereus, highly conserved proteins in Bacillus anthracis. J Biol Chem 280, 30856-30863.

Renault, P., Corthier, G., Goupil, N., Delorme, C. \& Ehrlich, S. D. (1996). Plasmid vectors for Gram-positive bacteria switching from high to low copy number. Gene 183, 175-182.

Redko, Y., Courtin, P., Mézange, C., Huard, C. \& Chapot-Chartier, M.-P. (2007). Lactococcus lactis gene $y j g B$ encodes a $\gamma$-D-glutaminyl-Llysyl-endopeptidase which hydrolyzes petidoglycan. Appl Environ Microbiol 73 (in press).

Sambrook, J., Fritsch, E. F. \& Maniatis, T. (1989). Molecular Cloning: a Laboratory Manual, 2nd edn. Cold Spring Harbor, NY: Cold Spring Harbor Laboratory.

Shockman, G. D. (1992). The autolytic ('suicidase') system of Enterococcus hirae: from lysine depletion autolysis to biochemical and molecular studies of the two muramidases of Enterococcus hirae ATCC 9790. FEMS Microbiol Lett 79, 261-267.

Shockman, G. D. \& Höltje, J. V. (1994). Microbial peptidoglycan (murein) hydrolases. In Bacterial Cell Wall (New Comprehensive Biochemistry), vol. 27, pp. 131-167. Edited by J.-M. Ghuysen \& R. Hackenbeck. Amsterdam, The Netherlands: Elsevier.

Smith, T. J., Blackman, S. A. \& Foster, S. J. (2000). Autolysins of Bacillus subtilis: multiple enzymes with multiple functions. Microbiology 146, 249-262.
Steen, A., Buist, G., Leenhouts, K. J., El Khattabi, M., Grijpstra, F., Zomer, A. L., Venema, G., Kuipers, O. P. \& Kok, J. (2003). Cell wall attachment of a widely distributed peptidoglycan binding domain is hindered by cell wall constituents. J Biol Chem 278, 23874-23881.

Steen, A., Buist, G., Horsburgh, G. J., Venema, G., Kuipers, O. P., Foster, S. J. \& Kok, J. (2005a). AcmA of Lactococcus lactis is an $\mathrm{N}$ acetylglucosaminidase with an optimal number of LysM domains for proper functioning. FEBS J 272, 2854-2868.

Steen, A., Palumbo, E., Deghorain, M., Cocconcelli, P. S., Delcour, J., Kuipers, O. P., Kok, J., Buist, G. \& Hols, P. (2005b). Autolysis of Lactococcus lactis is increased upon D-alanine depletion of peptidoglycan and lipoteichoic acids. J Bacteriol 187, 114-124.

Studier, F. W. \& Moffatt, B. A. (1986). Use of bacteriophage T7 RNA polymerase to direct selective high-level expression of cloned genes. $J$ Mol Biol 189, 113-130.

Towbin, H., Staehelin, T. \& Gordon, J. (1979). Electrophoretic transfer of proteins from polyacrylamide gels to nitrocellulose sheets: procedure and some applications. Proc Natl Acad Sci U S A 76, 4350-4354.

Vollmer, W. \& Tomasz, A. (2000). The $p g d A$ gene encodes for a peptidoglycan $\mathrm{N}$-acetylglucosamine deacetylase in Streptococcus pneumoniae. J Biol Chem 275, 20496-20501.

Wecke, J., Madela, K. \& Fischer, W. (1997). The absence of D-alanine from lipoteichoic acid and wall teichoic acid alters surface charge, enhances autolysis and increases susceptibility to methicillin in Bacillus subtilis. Microbiology 143, 2953-2960.

Edited by: M. Kleerebezem 